\title{
An Overview of Cloud Interoperability
}

\author{
Magdalena Kostoska*, Marjan Gusev*, and Sasko Ristov*† \\ *University Ss Cyril and Methodius, FCSE, Skopje, Macedonia \\ Email: \{magdalena.kostoska, marjan.gushev\}@ finki.ukim.mk \\ $\dagger$ University of Innsbruck, Innsbruck, Austria \\ Email: sashko@dps.uibk.ac.at
}

\begin{abstract}
Unlike the network TCP/IP's and OSI's layered structure of protocols, which allows the independence of protocols of different layers, as well as defining the upper layer protocols through the protocols of the lower layers, the cloud service layers are tightly dependent on each other. For example, an application of the SaaS layer can neither communicate nor exchange data with another application found on the same layer. The goal of this paper is to overview the cloud interoperability and to analyze it as a service model perspective. Several aspects and categories of cloud interoperability are analyzed in this paper.

Index Terms-Migration, interoperability, portability.
\end{abstract}

\section{INTRODUCTION}

A DOPTION of cloud rests largely on interoperability and standardization as they define the new age IT industry [1].

The main stakeholders (cloud providers and clients) have opposite motivations for cloud interoperability. The providers prefer vendor lock-in situations to keep the clients and ensure higher profits enabling more and more cloud features. On contrary, the clients would like freedom, and the ability to choose the provider that offers the highest quality of services they want. Therefore, the need for cloud interoperability is more initiated by the clients than the providers.

Cloud interoperability is closely linked with cloud portability [2]. Whenever one analyzes the process of porting data and applications from one cloud provider to another, then the cloud interoperability is the essential problem to be solved. An easy way to port data, application and platform is through transferring an image of the virtual machine (VM) between the providers that use the same cloud environment.

There are a lot of surveys about ongoing initiatives that address the cloud computing interoperability. Petcu [3] discusses the classical problem of too many different approaches by various vendors in the way they realize the interface to the cloud and offer cloud features.

The interoperability problems in cloud computing arise when the clients are trying to exchange data, applications and services between different cloud providers. The identified problems can be classified into the following categories: a) system, initiated by incompatible implementations of cloud virtualization; b) applications, including incompatible application programs and code, c) service, defined by the ability to use various services hosted on different clouds; d) data, initiated by different standards of data presentation. Data interoperability is mainly addressed by other computing areas, whereas, the cloud interoperability addresses mainly the system, application and service interoperability.

This paper observes the cloud interoperability on IaaS, PaaS and SaaS levels analyzing its context from the management, platform or application levels. An approach is introduced to analyze the cloud interoperability as a service model.

\section{BACKGROUND}

The concept of interoperability is not a new one. In the fields of information technology or systems engineering, it has been defined as the ability of two or more heterogeneous elements to not only exchange, but also use the exchanged information (interoperate). However, in the field of Cloud computing, the concept of interoperability is rather new and has recently been an active field of research. In this section we will define and explain all related concepts.

The interoperability can not be uniformly defined - there are very many different definitions which vary in technological aspects, and development frameworks, which can be more general or address only some standard details. Generally, the definition of interoperability depends on the context of its application.

IEEE describes the interoperability as a system or product feature to work with other systems or products without additional intervention of the client [4]. According to NIST [5], the cloud interoperability allows seamless exchange and use of data and services among various cloud infrastructure offerings and to use the data and services exchanged to enable them to operate effectively together.

Interoperability can be regarded as the ongoing process of ensuring that the systems, procedures and culture of an organization are managed in such a way as to maximize opportunities for exchange and reuse of information. It includes many areas with its characteristics [6]:

- Technical interoperability - development of standards of communication, transport and representation

- Semantic interoperability - the use of various different terms to describe similar concepts may cause problems in communication, execution of programmes and data transfers.

- Political/Human interoperability - the decision to make resources widely available has implications for organizations, their employees and end-users 


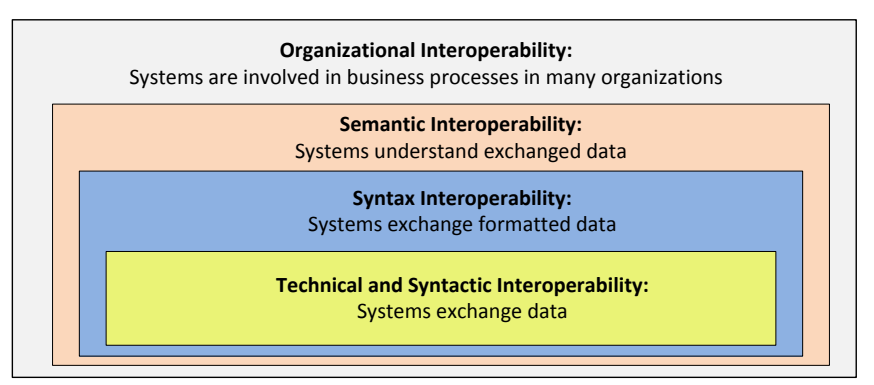

Fig. 1. Interoperability types and their correlation

- Interoperability of communities or societies - there is an increasing need to require access to information from a wide range of sources and communities.

- International interoperability - in international matters, there are variations in standard, communication problems, language barriers, differences in communication styles, and a lack of common basis.

In most known interoperability frameworks, this term is considered in three levels [7], as follows:

- Technical interoperability which includes standards and protocols. This aspect of interoperability covers the technical issues of linking computer systems and services.It includes key aspects such as open interfaces, interconnection services, data integration and middleware, data presentation and exchange, accessibility and security services [8]. Technical interoperability is usually associated with hardware/software components, systems and platforms that enable machine-to-machine communication to take place. This kind of interoperability is often centred on (communication) protocols and the infrastructure needed for those protocols to operate [9].

- Syntax interoperability is usually associated with data formats when they are exchanged among systems. Certainly, the messages transferred by communication protocols need to have a well-defined syntax and encoding, even if it is only in the form of bit-tables [9].

- Semantic interoperability is concerned with ensuring that the precise meaning of exchanged information is understandable by any other application that was not initially developed for this purpose. Semantic interoperability enables systems to combine received information with other information resources and to process it in a meaningful manner. Semantic interoperability is therefore a prerequisite for the front-end multilingual delivery of services to the client [8].

Additionally, organizational interoperability is also discussed, which allows systems to be involved in business processes of multiple organizations [8].

An overview of all interoperability types is given in Fig. 1. The application of interoperability in any domain is usually realized by defining and applying standards. Generally, the goal of interoperability and the standards is the same - to allow exchange and cooperation of computer services. Standards define protocol by which all service suppliers that implement the standards offer structured data and information exchange no matter the inner architectural design or implementation is used for the service.

\section{Cloud INTERoperability}

Interoperability in cloud can be considered and defined as a service model, and therefore, we will discuss interoperability of applications, platforms, and management.

Cloud application interoperability addresses the application components, whether they are deployed as IaaS, PaaS, or SaaS. An application component may be a complete monolithic application, or a service as a part of a distributed application. These components invoke respective platforms that implement various communications protocols and data presentation standards; and therefore, can not be used without cloud platform interoperability.

Cloud platform interoperability concerns the platform components, usually deployed as PaaS or IaaS. Information exchange and service discovery requires standard protocols to realize interoperable platforms.

Cloud management interoperability targets the management aspects between various cloud services deployed on SaaS, PaaS, or IaaS levels. Each provider realizes different cloud features and interfaces to manage them, so the clients would prefer to have a unique approach and generic off-the-shelf system management, offered via standard interfaces.

\section{A. IaaS level interoperability}

Interoperability on the IaaS level of cloud management implies simple and standardized management of infrastructures of different cloud systems. The management includes instantiating and control of virtual machines, enabling and discovering network characteristics, setting and editing security rules, etc.

This type of interoperability is the best defined when compared to other types. Fig. 2 shows a taxonomy of its basic concepts [10], [11], [12]:

- Access Mechanism - defines how a service in cloud may be accessed by users and/or software developers,

- Virtual Resources - service delivery as a complete software stack of installing a virtual machine,

- Network - addressing and API,

- Storage - management and organization of storage,

- Security - authentication, authorization, user accounts and encryption,

- Service-Level Agreement - architecture format, monitoring, and

- Other.

\section{B. PaaS level interoperability}

Interoperability on the PaaS level implies simple exchange of data and services among different platforms hosted on different infrastructures on cloud, and their effective reuse without extra effort on part of the user.

When analyzing the data exchange, one can consider data compatibility among different platforms, such as if numbers 


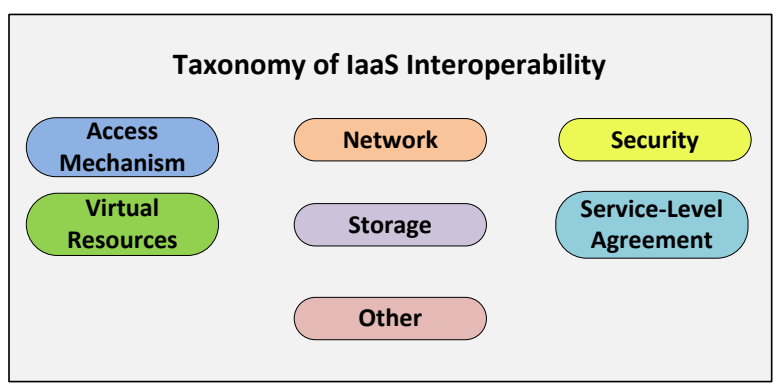

Fig. 2. Taxonomy of IaaS interoperability [10]

are to be transferred, then Little or Big Endian mode should be preserved, or a special function enabled to realize an easy transformation between the formats prior to transfer.

Analysis of the interoperability of services to be hosted in different cloud platforms rises the question of portability. For example, to transfer a service from one cloud to another that uses a different platform, initiates a lot of portability problems. If the origin and target clouds use the same environment, then a simple packing and copying procedure can be used to realize the porting process.

In case of different platforms on the origin and target clouds, one has to start a different transfer procedure that consists of packing, copying, instantiating, installing, deployment and customization to enable an interoperability. Still, there are open issues that address the way services interact with others, if there are additional cloud services invoked by the origin cloud that are not supported by the target cloud, or any dependence on a specific operating system hosted in the origin cloud.

\section{SaaS level interoperability}

Interoperability on the SaaS level of cloud applications implies simple exchange of data and services among different applications hosted on different platforms and infrastructures on cloud, and their effective reuse without extra effort on part of the user. Additionally, this type of interoperability can be considered from different application domains.

Interoperability on the level of applications is first defined by Kumar et al. [13] in 2010. According to their definition, interoperability can be considered in four categories (Fig. 3):

- Interoperability among applications in the same cloud,

- Data exchange and operation calls in applications on different cloud-computing environments

- Software programs that are distributed in different cloud environments and integrate data and applications in cloud in a unified way, and

- Migration of applications from one cloud environment to another.

When a client switches between two cloud providers on the SaaS level does not involve porting the applications and services, rather it involves exchange of structured data.

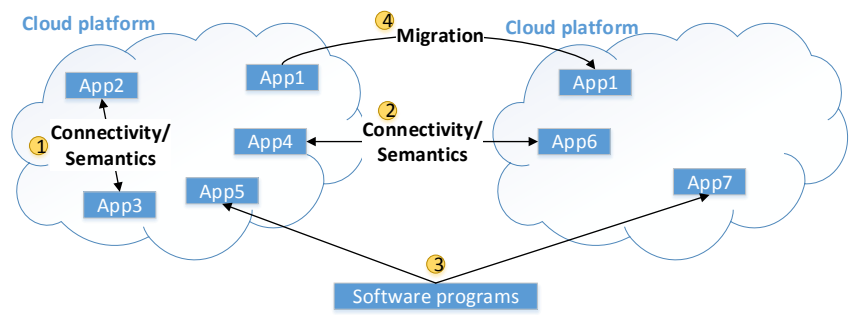

Fig. 3. Interoperability types in cloud applications [13]

TABLE I

DEVELOPMENT STATUS OF EACH ASPECT OF INTEROPERABILITY OF Cloud Computing

\begin{tabular}{lll}
\hline Context & Layer & Developing standards \\
\hline Management & IaaS & OCCI, CIMI, UCI \\
Platform & PaaS & Stub \\
Application & SaaS & mOSAIC \\
\hline
\end{tabular}

The cloud interoperability is probably the most significant to address the compatibility of exchanged data on the functional level, it means not just to transfer the structured data, but also all relations between them. So far, no interface has been developed to allow such an interoperability. The problem is mainly manifested on the definition on functional level of realization the interface to the application.

Most of the research in the area of SaaS level, and even on the PaaS level is limited by the support of the vendors. Usually, the vendors prefer to lock-in the customer to its cloud and do not cooperate in the efforts to support the interoperability on this level.

\section{DISCUSSION}

Table I presents the current development stage for each of the perspectives (i.e. categories), as well as, mapping of the use cases to the perspectives.

One can notice in Table I that certain aspects are more developed than other (i.e. interoperable management of virtual machines and application portability). We can also notice that the Platform context is least developed.

Large number of developing standards has arisen during the past few years:

- OCCI - The Open Cloud Computing Interface standard represents protocol and API for all kinds of IaaS management tasks [14]

- CIMI - Cloud Infrastructure Management Interface standard represents an interface for management of cloud services and the operations and attributes [15]

- UCI - Unified Cloud Interface concept aim to provide a unified interface for entire infrastructure stack using semantic technology [16]

- mOSAIC - The mOSAIC platform and engine enables deployment, configuration and management of applications using semantic technology [17] 
- OVF - Open Virtualization Format standard provides open and platform-independent packaging format for software solutions based on virtual systems [18]

- CAMP - Cloud Application Management for Platforms aims to standardizing cloud PaaS management API [19]

- TOSCA - The Topology and Orchestration Specification for Cloud Applications aims to standardize application description in order to provide portability and management [20]. We introduced the extension - PTOSCA, which handles several TOSCA weaknesses and ambiguities [21]. The demo applications for automated portability with P-TOSCA are developed for porting a SOA application [22] and an $N$-tier application [23].

- OData - The Open Data Protocol enables service creation to publish, share and edit resources via HTTP [24]

- CDMI - The Cloud Data Management Interface standard defines interface for creation, retrieval, update and deletion of data elements from the Cloud [25]

\section{CONCLUSION}

This paper gives an overview of the cloud interoperability on different service layers analyzed from cloud management, platform and application aspects as a service model. The cloud interoperability should be considered as cloud management on IaaS layer, exchange of data and services among different platforms (on PaaS layer) hosted on different infrastructures and exchange of structured data (on SaaS layer) among different applications deployed on platforms hosted on different infrastructures.

Although there are several standards and solutions on the data presentation level (data formats and communication protocols), still there are open issues on interoperability on systems and applications and there are no solutions when one wants to exchange structured data between providers. One can conclude that cloud interoperability on IaaS and PaaS levels has been addressed and several partial solutions exist, while the cloud interoperability on the SaaS level is still in an infant development.

\section{ACKNOWLEDGMENT}

This work was partially financed by the Faculty of Computer Science and Engineering at the "Ss. Cyril and Methodius" University, Skopje, Macedonia.

\section{REFERENCES}

[1] A. Parameswaran and A. Chaddha, "Cloud interoperability and standardization,” SETlabs briefings, vol. 7, no. 7, pp. 19-26, 2009.

[2] M. Kostoska, M. Gusev, and S. Ristov, "An overview of cloud portability," in Future Access Enablers for Ubiquitous and Intelligent Infrastructures, ser. Lecture Notes of the Institute for Computer Sciences, Social Informatics and Telecommunications Engineering, V. Atanasovski and A. Leon-Garcia, Eds. Springer International Publishing, 2015, vol. 159, pp. 248-254. ISBN 978-3-319-27071-5. [Online]. Available: http://dx.doi.org/10.1007/978-3-319-27072-2_32

[3] D. Petcu, "Portability and interoperability between clouds: challenges and case study," in Towards a Service-Based Internet. Springer, 2011, pp. 62-74. [Online]. Available: http://dx.doi.org/10.1007/978-3-642-24755-2_6
[4] IEEE, "610.7-1995 Standard Glossary of Computer Networking Terminology," Available online: http://ieeexplore.ieee.org/xpl/mostRecentIssue.jsp? punumber $=3284$.

[5] National Institute of Standards and Technology, "NIST cloud computing standards roadmap," Available online: http://www.nist.gov/itl/cloud/upload/NIST_SP-500-291_Version2_2013_June18_FINAL.pdf, Jul. 2013.

[6] UKOLN, "Looking at interoperability," Available online: http://www.ukoln.ac.uk/interop-focus/about/flyer-interoperability.pdf.

[7] H. Kubicek and R. Cimander, "Three dimensions of organizational interoperability," European Journal of ePractice, vol. 6, 2009.

[8] European Union, "European interoperability framework for Pan-European eGovernment services," Available online: http://ec.europa.eu/idabc/servlets/Docd552.pdf?id=19529.

[9] H. van der Veer and A. Wiles, "Achieving technical interoperability," European Telecommunications Standards Institute, 2008.

[10] Z. Zhang, C. Wu, and D. W. Cheung, "A survey on cloud interoperability: taxonomies, standards, and practice," ACM SIGMETRICS Performance Evaluation Review, vol. 40, no. 4, pp. 13-22, 2013. doi: 10.1145/2479942.2479945. [Online]. Available: http://doi.acm.org/10.1145/2479942.2479945

[11] R. Prodan and S. Ostermann, "A survey and taxonomy of infrastructure as a service and web hosting cloud providers," in Grid Computing, 2009 10th IEEE/ACM International Conference on. IEEE, 2009. doi: 10.1109/GRID.2009.5353074 pp. 17-25.

[12] B. P. Rimal, E. Choi, and I. Lumb, "A taxonomy and survey of cloud computing systems," in INC, IMS and IDC, 2009. NCM'09. Fifth International Joint Conference on, 2009. doi: 10.1109/NCM.2009.218 pp. $44-51$.

[13] B. Kumar, J. C. Cheng, and L. McGibbney, "Cloud computing and its implications for construction IT," in Computing in Civil and Building Engineering, Proceedings of the International Conference, vol. 30, 2010, p. 315 .

[14] Open Grid Forum, “OCCI, Open Cloud Computing Interface,” Available online: http://occi-wg.org/, 2011. [Online]. Available: http://occi-wg.org/

[15] Distributed Management Task Force, "Cloud infrastructure management interface (CIMI) model and RESTful HTTP-based protocol," Available online: http://dmtf.org/sites/default/files/standards/documents/ DSP0263_2.0.0c.pdf, Mar. 2015.

[16] Google, "Unified cloud," Available online: http://code.google.com/p/unifiedcloud/wiki/ UCI_Architecture, 2010.

[17] F. Moscato, R. Aversa, B. Di Martino, T. Fortis, and V. Munteanu, "An analysis of mosaic ontology for cloud resources annotation," in Computer Science and Information Systems (FedCSIS), 2011 Federated Conference on, Sept 2011, pp. 973-980.

[18] Distributed Management Task Force, "Open virtualization format specification version 2.1.0," Available online: http://www.dmtf.org/sites/ default/files/standards/documents/DSP0265_1.0.0.pdf, Jan. 2014.

[19] M. Carlson, M. Chapman, A. Heneveld, S. Hinkelman, D. JohnstonWatt, A. Karmarkar, T. Kunze, A. Malhotra, J. Mischkinsky, A. Otto et al., "Cloud application management for platforms," Available online http://cloudspecs. org/camp/CAMP-v1.0.pdf.

[20] R. Han, M. M. Ghanem, and Y. Guo, "Elastic-TOSCA: Supporting elasticity of cloud application in TOSCA," in CLOUD COMPUTING 2013, The Fourth International Conference on Cloud Computing, GRIDs, and Virtualization, 2013, pp. 93-100.

[21] M. Gusev, M. Kostoska, S. Ristov, and A. Donevski, "P-TOSCA portability of SOA applications," in Proceedings of 5th International Conference on Cloud Computing and Service Science (CLOSER), Lisbon, Portugal, 2015, pp. 71-78. [Online]. Available: http://closer. scitevents.org/?y=2015

[22] S. Ristov, M. Kostoska, and M. Gusev, "P-TOSCA portability demo case," in 2014 IEEE 3rd International Conference on Cloud Networking (IEEE CLOUDNET), 2014. doi: 10.1109/CloudNet.2014.6969002 pp. 269-271.

[23] M. Gusev, M. Kostoska, and S. Ristov, "Cloud P-TOSCA porting of N-tier applications," in Proceedings of the 22nd International TELFOR Forum, IEEE Conference Publications, 2014. doi: 10.1109/TELFOR.2014.7034559 pp. 935-938.

[24] OASIS, “Open Data Protocol (OData) 4.0," Feb. 2014, http://docs.oasisopen.org/.

[25] SNIA, "Cloud Data Management Interface (CDMI) v1.1.1," Available online: http://www.snia.org/sites/default/files/ CDMI_Spec_v1.1.1.pdf, Mar. 2015. 